\title{
Innere Schweinehunde
}

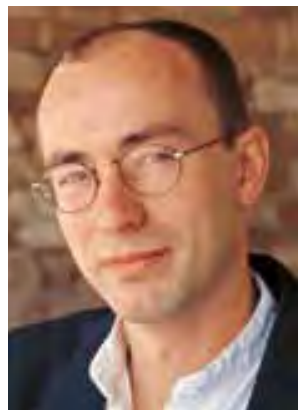

Dr. med. Dieter Schmid, Redaktionsleitung
Literatur:

[1] ].T. Gräsner et al. Einfluss der Basisreanimationsmaßnahmen durch Laien (...). Notfall + Rettungsmedizin 2012; doi: $10.1007 / \mathrm{s} 10049-012-1584-7$

[2] A. Bohn et al. Teaching resuscitation in schools: (...) Resuscitation 2012

83: 619-625; doi: 10.1016 j.resuscitation.2012.01.020
Die Zahl ist beschämend. Angenommen in Deutschland liegt eine Person auf dem Boden - ohne Atmung, blau angelaufen, die Augen verdreht. Wissen Sie, wie viele Menschen in einer solchen Situation stehen bleiben, um Wiederbelebungsmaßnahmen zu starten? Ganze 16 Prozent! Diese Rate nennt eine Studie der Deutschen Gesellschaft für Anästhesiologie (DGAI) [1]. Die Experten schätzen, dass pro Jahr bis zu 10.000 Menschen mehr gerettet werden könnten, wenn die Deutschen hier ein bisschen entschlossener wären. Besonders peinlich: Mit diesen 16 Prozent stehen wir im internationalen Vergleich außerordentlich schlecht da. In Skandinavien beginnen laut DGAI zum Beispiel 60 Prozent der Passanten eine Reanimation, wenn ein Mensch einen Kreislaufstillstand erleidet. Woran liegt es, dass so viele Deutsche es nicht hinkriegen, in einer solchen Situation ihren „inneren Schweinehund“ zu überwinden? Ich hielte es für ausgemachten Blödsinn, diesen Sachverhalt einem in den Medien oftmals beklagten Mangel an Zivilcourage zuzuschreiben. Meiner Ansicht nach sind wir nicht ängstlicher oder mutiger als andere Völker. Schaut man genauer hin, erkennt man rasch, dass die wahre Ursache, wie bei vielen Problemen, in einem Mangel an Bildung und Aufklärung liegt. So ist in Skandinavien das Thema Reanimation zum Beispiel auch in den Schulen präsent. Und Studien zeigen, das sich auch in Deutschland unter Schülern die Bereitschaft zur Beatmung und Herzdruckmassage dramatisch steigern ließe, wenn im Unterricht die Grundlagen der Wiederbelebung geübt würden [2]. Kein Wunder: Denn wer weiß, wie's geht, hat auch weniger Angst, etwas falsch zu machen. Das gilt natürlich auch für junge Mediziner. Deswegen erläutern wir Ihnen in diesem Heft ausführlich die aktuellen Leitlinien zum „Basic and Advanced Life Support“ (BLS und ALS). Im Artikel „Rhythm of Life“ auf S. 38 finden Sie alles, was Sie brauchen, wenn Sie Menschen mit Herz-Kreislauf-Stillstand ins Leben zurückholen möchten.

Um „innere Schweinhunde“ ganz anderer Natur geht es im „Facharzt-Check: Psychiatrie“ auf S. 18. Die Ärzte in diesem Fach kämpfen gegen Krankheiten der fiesesten Sorte nämlich die Leiden der Seele. Dabei räumt dieser Artikel mit einem weit verbreiteten Vorurteil auf: Psychiatrie ist keineswegs „bloßes Gerede“. Wer Wahngedanken, Depressionen und Ängste behandeln möchte, muss auch das „Soma“ seiner Patienten im Blick haben! Denn mitunter steckt hinter einer Depression eine Borreliose, hinter Angstattacken ein Phäochromozytom - und wenn ein Alkoholkranker delirant wird, braucht man notfallmedizinische Kenntnisse.

Mein Wunsch so kurz vor der Adventszeit: Mögen sich sämtliche „Schweinehunde“ - egal welcher Natur - von Ihnen fernhalten! Ich wünsche Ihnen eine friedliche Vorweihnachtszeit! Herzlichst, Ihr

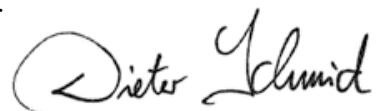

\section{Liebe auf Distanz - funktioniert das?}

Viele Studenten führen eine Fernbeziehung. Junge Mediziner trifft's besonders oft. Denn sie müssen für ihr Studium häufiger als Studierende anderer Fächer in eine neue Stadt wechseln (S. 14). Für eine junge Liebe ist so eine Trennung eine ziemliche Belastungsprobe. Wie schafft man trotz der großen Distanz Nähe? Wie baut man Vertrauen auf und entwickelt gemeinsame Perspektiven? Langzeitstudien zeigen: Nach zwei bis drei Jahren ist meistens Schluss mit der Fernbeziehung. Viele Paare trennen sich. Einige schaffen aber auch das
Happy End und ziehen zusammen. Medizinstudenten haben da ein Problem: Für sie ist ein Wohnortwechsel nach drei Jahren oft gar nicht möglich. Sollte man bei diesen Aussichten lieber gleich die Segel streichen? Oder ist die Liebe auf Distanz zu unrecht als Liebeskiller verschrien?

- Glauben Sie, dass eine Fernbeziehung parallel zum Medizinstudium funktionieren kann?

Geben Sie Ihr Statement ab und kommentieren Sie unter: http://bit.ly/RZ33os
- Ergebnis der Umfrage in Via medici 4.12: Wir fragten, ob Sie Ärzten erlauben würden, eine Zwangsbehandlung auch ohne richterliche Kontrolle anzusetzen. Eine haarscharfe Mehrheit von $53 \%$ ist dagegen und der Meinung, „dass Ärzte nicht über dem Recht stehen und jeder Eingriff in so substanzielle Persönlichkeitsbereiche wie Zwangsmaßnahmen kontrolliert werden sollte“. 46\% der Teilnehmer sind hingegen der Meinung, dass es Medizinern erlaubt sein sollte, Zwangsbehandlungen eigenverantwortlich anzuordnen. Mehr Kommentare unter: .../aktuelles/aktion/miniumfrage4-12.html 